\title{
A COMUNIDADE ZOOPLANCTÔNICA E SEU USO COMO BIOINDICADORA DO ESTADO TRÓFICO DE UMA REGIÃO DO RESERVATÓRIO DA UHE DE FURNAS
}

\author{
Tiago Rosa de Souza ${ }^{1}$ \\ Marcos Vinícius Nunes ${ }^{2}$ \\ Lidiane Cristina da Silva ${ }^{3}$
}

RESUMO: A comunidade zooplanctônica em águas doces é composta por um conjunto de organismos exclusivamente heterótrofos responsáveis pela transferência de matéria e energia entre os níveis tróficos inferiores da cadeia (fitoplâncton) e níveis maiores (peixes, pequenos invertebrados). Muitas das espécies são utilizadas como bioindicadoras já que possuem grande sensibilidade ambiental. $O$ estudo teve como objetivo analisar a comunidade zooplanctônica em três pontos do reservatório da UHE de Furnas localizados próximo ao município de Alfenas (MG) visando utilizar a comunidade como bioindicadora do estado trófico do ambiente. Foram aferidas as principais variáveis ambientais in situ e em laboratório (concentrações de nutrientes, $\mathrm{pH}$, temperatura, condutividade e oxigênio dissolvido). Amostras de zooplâncton foram coletadas por arrastos verticais através de uma rede de plâncton com malha de $68 \mu \mathrm{m}$. O número de espécies registradas foi considerado baixo, característico de corpos d'água pobres em nutrientes e com baixos recursos alimentares. No ponto $\mathrm{P} 3$ foi registrado o maior número de táxons, maior diversidade e equitabilidade de espécies, porém, menores densidades numéricas. Já nos

\footnotetext{
${ }^{1}$ Graduando em Ciências Biológicas, Universidade do Vale do Sapucaí. Trs-souza@hotmail.com

${ }^{2}$ Biólogo, Programa de Pós-Gradução em Ecologia e Recursos Naturais, Universidade Federal de São Carlos.vnnybio@hotmail.com

${ }^{3}$ Bióloga, Programa de Pós-Gradução em Ecologia e Recursos Naturais, Universidade Federal de São Carlos. lidianecrsilva@gmail.com
} 
pontos $\mathrm{P} 1$ e $\mathrm{P} 2$, onde a riqueza, diversidade e equitabilidade de espécies foram menores, a dominância e as densidades numéricas registradas foram altas. Isso demonstra 0 aumento de perturbação ambiental nos pontos $\mathrm{P} 1$ e P2 em relação ao P3, para os quais também foram registrados maiores valores de nutrientes. A comunidade zooplactônica da região amostrada no reservatório pode ser considerada como bioindicadora dos parâmetros ambientais do ecossistema, podendo ser observadas diferenças na riqueza, diversidade e abundância das espécies dessa comunidade entre os pontos amostrados.

Palavras-chave: Zooplâncton. Eutrofização. Biodiversidade

\section{INTRODUÇÃO}

A comunidade zooplanctônica em águas doces é composta por um conjunto de organismos pertencentes a diversos grupos taxonômicos, como Cladocera, Copepoda, Rotifera e Protozoa. É uma das divisões do plâncton composta por organismos exclusivamente heterótrofos responsáveis pela transferência de matéria e energia produzida pelas microalgas (fitoplâncton) servindo de alimento para inúmeros organismos, desde pequenos invertebrados, peixes (muitos de valor comercial) até grandes mamíferos, como as baleias e possui grande importância ecológica nos ambientes aquáticos (SIPAÚBA-TAVARES \& ROCHA, 2001).

O zooplâncton distribui-se de forma não homogênea em seu habitat e exibe padrões diferentes de segregação espacial, os quais, às vezes, podem sofrer alterações no decorrer de algumas horas (PINTO-COELHO, 2003). A comunidade possui ainda movimentos de migração vertical tanto em ambientes marinhos como de água doce, e depende de vários fatores tais como local, estação, idade e sexo dos organismos (COSTA, 1998)

Segundo Esteves (1998) o zooplâncton possui um papel central na dinâmica de um ecossistema aquático, abrangendo diversos aspectos. Por exemplo, regiões com altas densidades de zooplâncton (sobretudo crustáceos copépodos) podem ser consideradas bons locais para pesca devido às maiores possibilidades do desenvolvimento das espécies de peixes. Outro fato relevante que o autor menciona é que muitas das espécies 
zooplanctônicas são utilizadas como indicadoras hidrológicas, ou seja, as espécies têm preferências (por razões fisiológicas) por diferentes tipos de águas (com determinada temperatura, concentração de nutrientes, teor de oxigênio, etc).

Segundo Coelho-Botelho (2000) o estudo da comunidade zooplanctônica possui um papel imprescindível no biomonitoramento dos ecossistemas aquáticos por apresentar grande sensibilidade ao ambiente e responde a quaisquer tipos de impactos seja ele quantitativo e qualitativo a qual se refere a composição e diversidade da comunidade.

Assim uma alteração mesmo minuciosa destas variáveis pode afetar drasticamente a sobrevivência das espécies desencadeando um desequilíbrio na cadeia trófica, modificando principalmente a riqueza e a abundância dessa comunidade.

Dessa forma, o estudo teve como objetivo analisar a diversidade e abundância da comunidade zooplanctônica em três pontos do reservatório da UHE de Furnas visando o conhecimento desta relacionado à qualidade da água e o estado trófico do ambiente.

\section{1. ÁREA DE ESTUDO}

Reservatórios geralmente são sistemas multicompartimentais e de grande complexidade espacial e temporal. Grande parte da variabilidade intra reservatório ocorre ao longo do eixo principal, sendo determinada por gradientes longitudinais de velocidade de fluxo, tempo de retenção, profundidade, largura, transparência e penetração de luz, estratificação térmica, concentração de partículas em suspensão e de nutrientes (THORNTON et al.,1990; TUNDISI et al., 1993; NOGUEIRA et al., 1999).

O reservatório da Usina Hidrelétrica de Furnas está situado na Bacia do rio Grande, ao Sul do estado de Minas Gerais (46 $16^{\circ} \mathrm{W}, 20^{\circ} 40^{\prime} \mathrm{S}$ ). É formado pelos rios Grande (Braço Norte) e Sapucaí (Braço Sul). A construção do mesmo teve como ocorrência no ano de $1963 \mathrm{com}$ intuito de produzir energia elétrica, mas hoje a população também usufrui do reservatório como área de lazer, turismo e subsistência através de atividades como comercio, pesca e cultivo de peixes, abrangendo cerca de 30 cidades, entre estas, a cidade de Alfenas, região onde foi realizado o presente estudo (RELATÓRIO UHE DE FURNAS, 2004).

O reservatório da UHE de Furnas é o maior reservatório da região Sudeste do Brasil, apresenta extensão máxima de $220 \mathrm{Km}$ com perímetro de $3500 \mathrm{Km}$ e área inundada 
de $1440 \mathrm{Km}^{2}$, chegando a um volume total de 22,95 bilhões $\mathrm{m}^{3}$ de água. $\mathrm{O}$ tempo de residência da água é de aproximadamente 160 dias e sua profundidade máxima é $90 \mathrm{~m}$ (SÁ-JUNIOR, 1994; FURNAS, 2004).

\section{MATERIAL E MÉTODOS}

As amostragens foram realizadas em abril de 2013 no reservatório da UHE de Furnas localizado próximo ao município de Alfenas (MG) em três pontos, cujas

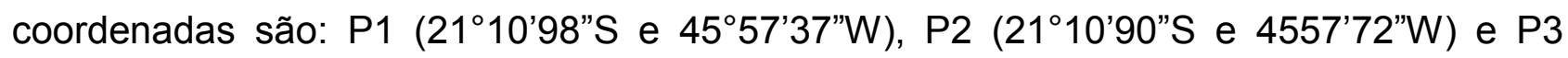

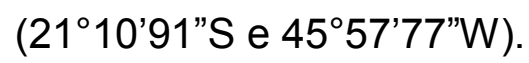

Em cada ponto de amostragem foram determinadas in situ as variáveis limnológicas (condutividade, oxigênio dissolvido, $\mathrm{pH}$ e temperatura) por meio do multisensor Horiba U10. Também foram coletadas amostras de água para a determinação das concentrações de nutrientes, entre estes: Nitrogênio e Fósforo total (VALDERRAMA, 1981) e Silicato (GOLTERMAN et al.,1978),

As amostras de zooplâncton foram coletadas com auxílio de rede de plâncton com 68 micrômetros de abertura de malha e de $25 \mathrm{~cm}$ de diâmetro. As amostras foram transferidas para frascos de polietileno, preservadas com formol a 4\%. A identificação dos organismos foi feita utilizando-se bibliografia especializada (KOSTE, 1978; REID, 1985; ELMOOR-LOUREIRO, 1997; SANTOS-SILVA, 2000; SILVA, 2003).

As quantificações dos microcrustáceos (Copepoda e Cladocera) foram feitas em placas de acrílico quadriculadas sob microscópio estereoscópico com aumento de até 50 vezes. Para os rotíferos e protozoários, subamostras de $1 \mathrm{ml}$ foram quantificadas em câmara de Sedgewick-Rafter, sob microscópio óptico com aumento de até 1000 vezes.

Para a comparação da diversidade de espécies entre os pontos amostrados foram calculados: Riqueza taxonômica; índice de Diversidade de Shannon-Wiener (H') (SHANNON \& WEAVER, 1949) e índice de Dominância.

Também foi determinada a similaridade de Bray-Curtis entre a densidade dos organismos da comunidade zooplanctônica nos pontos de amostragem. $O$ índice de similaridade foi calculado utilizando o programa Past-Paleontological Statistics Software (HAMMER et al., 2001). 
Para verificar possíveis correspondências entre os parâmetros ambientais e a densidade dos grupos da comunidade zooplanctônica foi realizada a análise de correspondência canônica (CCA), através do programa CANOCO 3.12 (TER BRAAK \& SMILAUER, 2002).

\section{RESULTADOS E DISCUSSÕES}

$\mathrm{Na}$ tabela I estão expressos os valores das variáveis limnológicas e das concentrações dos nutrientes dos pontos amostrados. Pode-se observar que não houve grande variação nos valores de $\mathrm{pH}$, condutividade, temperatura e oxigênio dissolvido. Em relação às concentrações de nutrientes foram registradas em alguns pontos valores mais elevados em relação aos demais como no ponto $\mathrm{P} 1$, local onde foram registradas as maiores concentrações de nitrogênio total $\left(122.96 \mu \mathrm{gL}^{-1}\right)$, nitrato $\left(50.93 \mu \mathrm{gL}^{-1}\right)$ e amônio $\left(67.18 \mu \mathrm{gL}^{-1}\right)$ e no P2 com a maior concentração de nitrito $\left(0.30 \mu \mathrm{gL}^{-1}\right)$.

No geral esses valores são típicos de ambientes não perturbados, demonstrando que essa região do reservatório da UHE de Furnas ainda é uma localidade cujas características se aproximam da oligotrofia (baixos valores de nutrientes).

Tabela I - Variáveis limnológicas e concentrações dos nutrientes da água registrados nos três pontos amostrados no reservatório da UHE de Furnas.

\begin{tabular}{c|c|c|c}
\hline Variáveis & P1 & P2 & P3 \\
\hline $\mathrm{pH}$ & 6,90 & 6,79 & 7,42 \\
\hline Condutividade $\left(\mu \mathrm{S} . \mathrm{cm}^{-1}\right)$ & 34 & 32 & 33 \\
\hline Temperatura $\left(\mathrm{C}^{\circ}\right)$ & 24,07 & 25,43 & 25,90 \\
\hline Oxigênio dissolvido $\left({\left.\mathrm{mg} . \mathrm{L}^{-1}\right)}^{\prime}\right.$ & 7,91 & 7,52 & 7,48 \\
\hline Fósforo Total $\left(\mu \mathrm{gL}^{-1}\right)$ & 31,40 & 31,08 & 29,77 \\
\hline Fosfato Total Dissolvido & 9,73 & 12,79 & 12,48 \\
\hline Fosfato Orgânico $\left(\mu \mathrm{gL}^{-1}\right)$ & 3,23 & 4,23 & 4,22 \\
\hline Fosfato Inorgânico $\left(\mu \mathrm{LL}^{-1}\right)$ & 6,51 & 8,56 & 8,27 \\
\hline Nitrogênio Total $\left(\mu \mathrm{gL}^{-1}\right)$ & 122,96 & 89,69 & 64,91 \\
\hline Nitrito $\left(\mu \mathrm{gL}{ }^{-1}\right)$ & 0,13 & 0,30 & 0,07 \\
\hline Nitrato $\left(\mu g \mathrm{~L}^{-1}\right)$ & 50,93 & 23,54 & 15,99 \\
\hline
\end{tabular}




\begin{tabular}{c|c|c|c}
\cline { 1 - 1 } Amônio $\left(\mu \mathrm{gL}^{-1}\right)$ & 67,18 & 46,97 & 40,75 \\
\hline Silicato $\left(\mu \mathrm{gL}^{-1}\right)$ & 4,34 & 7,43 & 5,49 \\
\hline
\end{tabular}

Pelas análises qualitativas das amostras foi registrado um total de 13 táxons para a comunidade zooplanctônica. Para o P3 (Tabela II) foi registrado a maior riqueza taxonômica em relação aos demais, com 8 táxons. Trichocerca cappucina e Asplanchna sieboldi representantes do grupo Rotifera, além das fases juvenis de copepoditos de Calanoida e Cyclopoida (Copepoda) foram os mais freqüentes nas amostras do reservatório, ocorrendo em dois ou nos três pontos de amostragem.

O número de espécies registradas foi considerado baixo, característico de corpos d'água pobres em nutrientes e com baixos recursos alimentares. O maior número de táxons registrado no ponto 3 provavelmente se deve à presença de macrófitas verificada durante a amostragem. As presenças dessas espécies de macrófitas possibilitam melhores condições alimentares e um maior número de micro-habitats para as espécies zooplanctônicas do local, proporcionando vantagens adaptativas e melhores condições de sobrevivência (SANTOS-WINIEWSKI, 1998). Espécies típicas de ambientes litorâneos e com presença de macrófitas foram registradas no ponto $\mathrm{P} 3$, representantes da família Chydoridae (Cladocera) como as espécies Alona glabra e Coronatella poppei.

Tabela II - Composição e riqueza taxonômica da comunidade zooplanctônica encontrada nos três pontos amostrados na represa de Furnas em Barranco Alto - MG.

\begin{tabular}{l|c|c|c}
\hline Táxons & P1 & P2 & P3 \\
\hline Cladocera & & & \\
\hline Alona glabra & & & $\mathrm{X}$ \\
\hline Alona guttata & & $\mathrm{X}$ & \\
\hline Coronatela poppei & & & $\mathrm{X}$ \\
\hline Diaphanosoma spinulosum & & & $\mathrm{X}$ \\
\hline Copepoda & & & \\
\hline Cyclopoida & & & \\
\hline Eucyclops serrulatus & & $\mathrm{X}$ & \\
\hline
\end{tabular}




\begin{tabular}{l|c|c|c}
\hline Copepodito de Cyclopoida & & $\mathrm{X}$ & $\mathrm{X}$ \\
\hline Náuplios de Cyclopoida & & $\mathrm{X}$ & \\
\hline Calanoida & & & \\
\hline Notodiaptomus cearensis & & $\mathrm{X}$ & \\
\hline Copepodito de Calanoida & $\mathrm{X}$ & $\mathrm{X}$ & $\mathrm{X}$ \\
\hline Rotifera & & & \\
\hline Asplanchna sieboldi & $\mathrm{X}$ & & $\mathrm{X}$ \\
\hline Trichocerca cappucina & $\mathrm{X}$ & & $\mathrm{X}$ \\
\hline Bdelloidea & & $\mathrm{X}$ & $\mathrm{X}$ \\
\hline Protozoários & & & \\
\hline Arcella vulgaris & $\mathrm{X}$ & & \\
\hline Arcella megastoma & & $\mathrm{X}$ & \\
\hline Riqueza taxonômica & $\mathbf{4}$ & $\mathbf{5}$ & $\mathbf{8}$ \\
\hline
\end{tabular}

Pelas análises quantitativas verifica-se que o $\mathrm{P} 1$ foi o ponto que registrou a maior densidade de indivíduos com 122,5 ind. $\mathrm{m}^{-3}$ e os táxons para os quais foram registrados os maiores valores foram Trichocerca cappucina e Asplanchna sieboldi no ponto P1 e Arcella mesgastoma no P2, todos com 60 ind. $\mathrm{m}^{-3}$ (Tabela III e Figura 1).

Através dos índices calculados para a comunidade zooplanctônica pode ser verificado que o maior valor de diversidade foi registrado no P3 com 2,02 bits. Ind. ${ }^{-1}$. Já o maior valor de dominância de espécies foi registrado no P2 com 0,79. Além do maior valor de diversidade, alto valor de equitabilidade também foi registrado no P3 com 0,97. Para esse índice o menor valor foi registrado no P2 com 0,26 (Figura 2).

No ponto P3 foi registrado o maior número de táxons, maior diversidade e equitabilidade de espécies, porém, menores densidades numéricas. Já nos pontos P1 e P2, onde a riqueza, diversidade e equitabilidade de espécies foram menores, a dominância e as densidades numéricas registradas foram altas. Isso demonstra 0 aumento de perturbação ambiental nos pontos P1 e P2 em relação ao P3, para os quais também foram registrados maiores valores de nutrientes. Isso pode ter influenciado no aumento do número de indivíduos em detrimento do número de espécies. 
Tabela III - Densidade de indivíduos (ind. $\mathrm{m}^{-3}$ ) da comunidade zooplanctônica registrada nos três pontos amostrados do reservatório da UHE de Furnas.

\begin{tabular}{l|c|c|c}
\hline Táxons & P1 & P2 & P3 \\
\hline Cladocera & & & \\
\hline Alona glabra & & & 0,75 \\
\hline Alona guttata & & & 1,5 \\
\hline Coronatela poppei & & & 1,5 \\
\hline Diaphanosoma spinulosum & & & \\
\hline Copepoda & & & \\
\hline Cyclopoida & & 1 & \\
\hline Eucyclops serrulatus & & 0,5 & 0,75 \\
\hline Copepodito de Cyclopoida & & 1,5 & \\
\hline Náuplios de Cyclopoida & & & \\
\hline Calanoida & & 0,5 & \\
\hline Notodiaptomus cearensis & 0,75 & 0,75 & 1,5 \\
\hline Copepodito de Calanoida & & & \\
\hline Rotifera & 60 & & 0,75 \\
\hline Trichocerca cappucina & 60 & & 0,75 \\
\hline Asplanchna sieboldi & & 1,75 & 0,75 \\
\hline Bdelloidea & & & \\
\hline Protozoa & 1,75 & & \\
\hline Arcella vulgaris & & 60 & \\
\hline Arcella megastoma & & $\mathbf{6 7 , 5}$ & $\mathbf{8 , 2 5}$ \\
\hline Densidade total & &
\end{tabular}




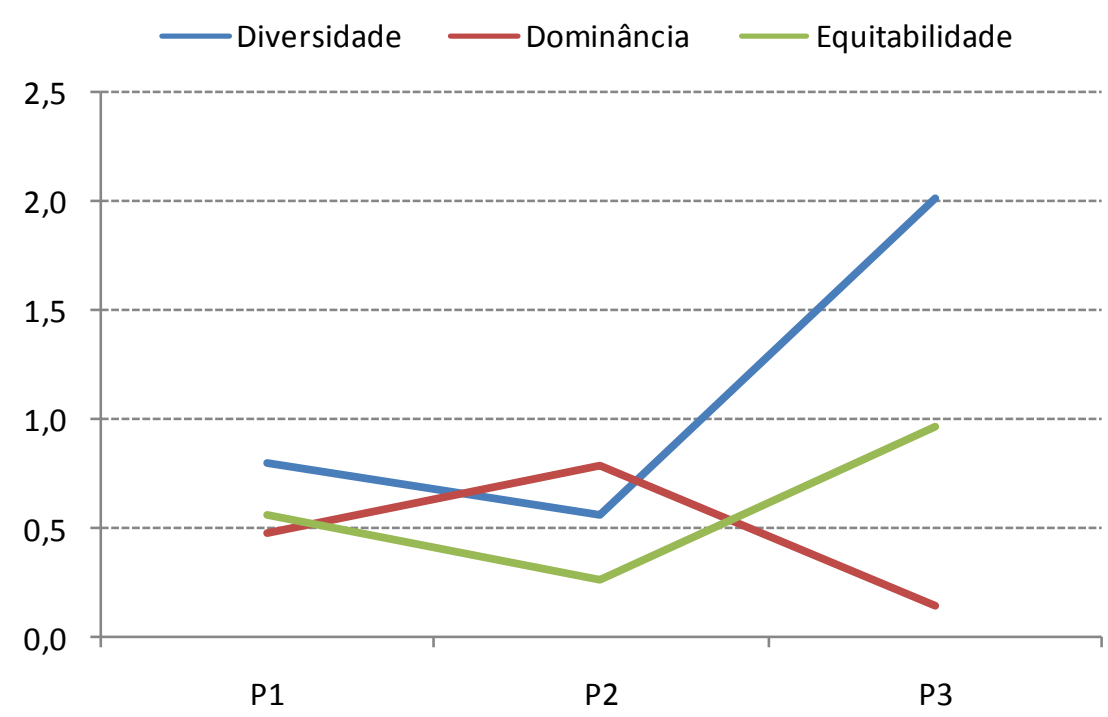

Figura 2 - Valores dos índices de diversidade, dominância e equitabilidade, obtidos nos três pontos amostrados no reservatório da UHE de Furnas.

Geralmente em ambientes mais perturbados (com maiores concentrações de nutrientes) há maiores abundâncias de uma ou poucas espécies e estas, na maioria, são de pequeno porte, como os Rotifera e Protozoa (MATSUMURA-TUNDISI et al.,1990) Essa associação também foi verificada no reservatório da UHE de Furnas, já que no ponto P1 foram registradas altas densidades para as espécies Asplanchna sieboldi e Trichocerca cappucina, representantes do grupo Rotifera.

Nos pontos $\mathrm{P} 1$ e P2 pode ser notada a influência antrópica na região já que na margem oposta desses pontos há uma plantação de banana, que pode acarretar sérios problemas para a biota local devido à lixiviação de agrotóxicos. Provavelmente essa é a causa das maiores concentrações de nutrientes nesses dois pontos de amostragem.

Através do dendograma de similaridade de Bray-Curtis (Figura 3) foi possível notar que praticamente não existe similaridade entre os pontos amostrados, sendo que os pontos que apresentaram maior semelhança entre si foram os pontos P2 e P3 com aproximadamente $10 \%$. Essa baixa similaridade se deve à baixa semelhança entre os táxons representativos de cada ponto e ainda às suas diferentes abundâncias registradas.

A Análise de Componentes Principais (CCA) aplicada entre os valores de densidade numérica e das variáveis ambientais do reservatório da UHE de Furnas 
apontou correlações interessantes. Nota-se que os pontos P1 e P2 estiveram correlacionados às variáveis que caracterizam possíveis impactos, caso estejam em valores extremos, como a condutividade elétrica e os nutrientes (fósforo e nitrogênio). $\mathrm{O}$ ponto P3 foi diferenciado dos demais, sendo correlacionado aos valores de $\mathrm{pH} e$ temperatura da água. Nota-se ainda que as espécies para as quais foram registradas as maiores densidades (Asplanchna sieboldi, Trichocerca cappucina e Arcella megastoma) também estiveram associadas aos parâmetros que caracterizam maiores trofias do ambiente (condutividade, nutrientes e oxigênio). As espécies de Copepoda, assim como Arcella vulgaris, os náuplios de Cyclopoida e Alona guttata também estiveram associadas a essas variáveis. A maioria das espécies do grupo Cladocera, as fases juvenis de copepoditos do grupo Copepoda e a Ordem Bdelloidea do grupo Rotifera foram associados à temperatura da água e aos valores de $\mathrm{pH}$. Provavelmente esses indivíduos são os mais sensíveis em relação às concentrações de nutrientes e às alterações na concentração de oxigênio dissolvido e na condutividade elétrica da água.

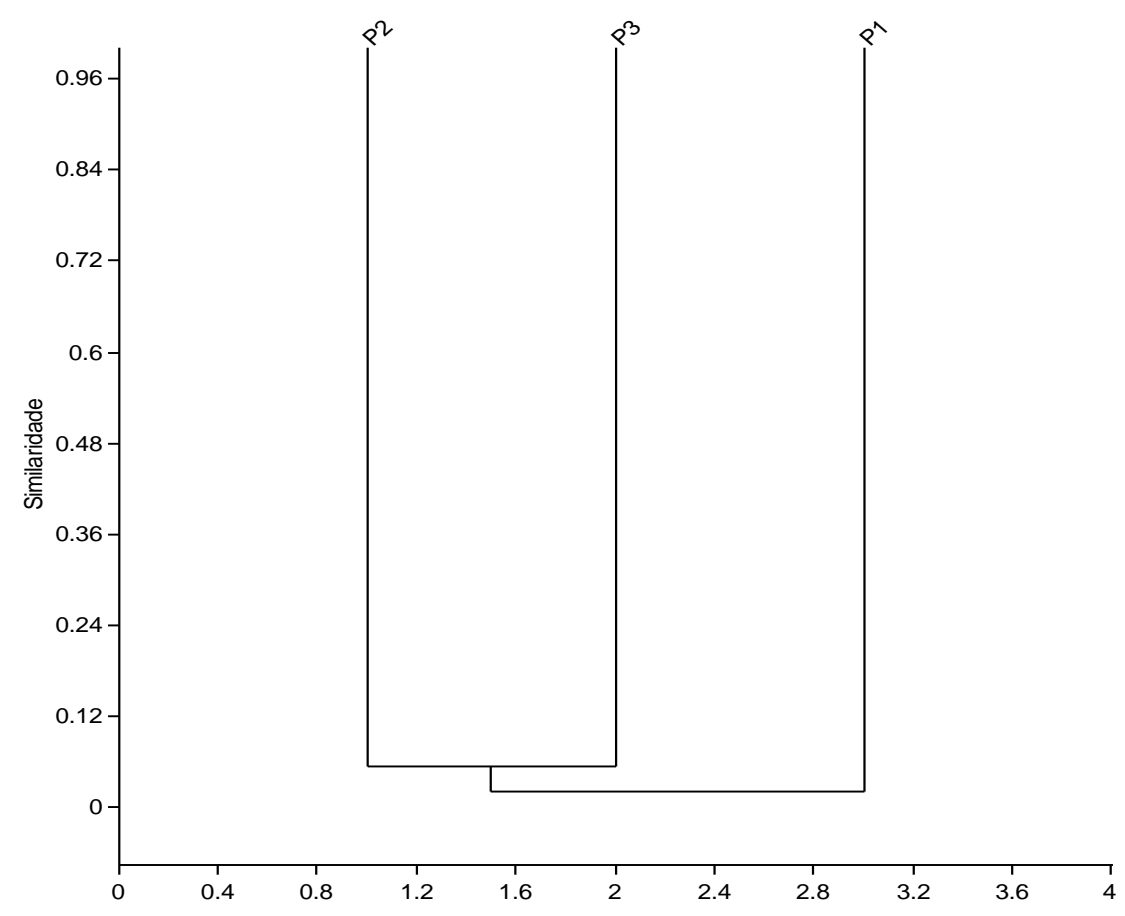

Figura 3 - Dendograma de similaridade de Bray-Curtis entre os três pontos amostrados no reservatório da UHE de Furnas. 


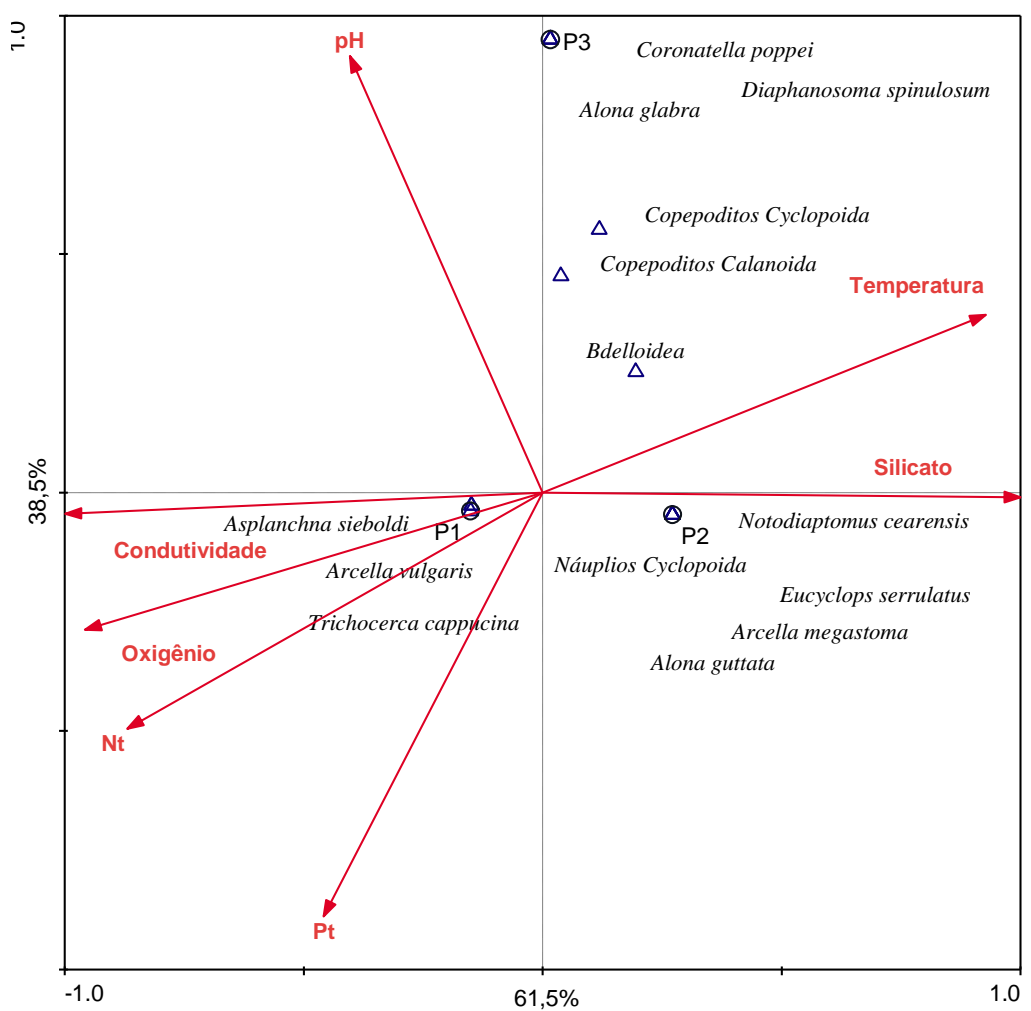

Figura 4. Análise de Componentes Principais (CCA) aplicada às densidades das espécies zooplanctônicas e às variáveis ambientais do reservatório da UHE de Furnas.

\section{CONCLUSÕES}

A comunidade zooplactônica da região amostrada no reservatório da UHE de Furnas pode ser considerada como bioindicadora dos parâmetros ambientais do ecossistema, podendo ser observadas diferenças na riqueza, diversidade e abundância das espécies dessa comunidade entre os pontos amostrados.

\section{REFERÊNCIAS BIBLIOGRÁFICAS}

COELHO-BOTELHO, M. J. Dinâmica da comunidade zooplanctônica e sua relação com o grau de trofia em reservatórios. Universidade Federal de São Carlos, 2000. Disponível 
em: <www.biota.org.br/inf/histórico/simp2003/textos/zooplâncton.pdf> acesso em: 24 setembro 2013.

COSTA, A.R.G. Migração vertical diurna e teores de lipídeos do zooplâncton do reservatório do reservatório da Pampulha $(\mathrm{BH} / \mathrm{MG})$ e do reservatório de Furnas (Passos/MG). Dissertação (Mestrado em Ecologia, Conservação e manejo da Vida Silvestri), Instituto de Ciências Biológicas, Universidade Federal de Minas Gerais, Belo Horizonte, 1998, $159 \mathrm{f}$.

ELMOOR-LOUREIRO, L.M. .Manual de identificação de Cladóceros límnicos do Brasil. Brasilia: Editora Universa, 156 p., 1997.

ESTEVES, F. A. 1998. Fundamentos de Limnologia. Rio de Janeiro : Interciência, FINEP, $575 \mathrm{p}$.

GOLTERMAN, H.L.; CLYMO, R.S.; OHNSTAD, M. A. M.Methods for physical and chemical analysis of freshwaters.2 ed. Oxford: Blackwell Scientific Publications, 213p., 1978.

HAMMER, O.; HARPER, D. A. T.; RYAN, P. D. Past: Palaeontological Statistc Software package for education and data analysis. Palaeontologia Electronica, 4 (1): 9. 2001.

KOSTE, W. Rotatoria die radertiere mitteleuropas, Übeiordnung Monogononta. Berlim: Gebriider Bernträger, 1010 p., 1978.

MATSUMURA-TUNDISI, T.; LEITÃO, S. N.; AGUENA, L. S.; MIYAHARA, J. Eutrofização da Represa de Barra Bonita: estrutura e organização da comunidade de Rotifera. Rev. Bras. Biol. vol. 50 (4), p. 923-935, 1990.

NOGUEIRA, M.G.; HENRY, R.; MARICATTO, F.E. Spatial and temporal heterogeneity in 
the Jurumirim Reservoir, São Paulo, Brazil.Lak. Reserv. Res. Manag., v. 4, p.107-112. 1999.

PINTO-COELHO, R.M. Métodos de coleta, preservação, contagem e determinação de biomassa em zooplâncton de águas epicontinetais. In: BICUDO, C.E.M.; BICUDO, D.C. Amostragem em limnologia. São Cartos:RiMa. p. 149-165. 2003.

RELATÓRIO UHE DE FURNAS. Furnas Centrais Elétricas S. A. Belo Horizonte: Plâncton Consultoria LTDA, 110p., 2004.

REID, J.W. Chave de identificação para as espécies continentais sulamericanas de vida livre da Ordem Cyclopoida (Crustacea, Copepoda). Bol. Zool., n.9, p. 17-143, 1985.

SÁ-JUNIOR, W. P. Production of planktonic biomass for feed alevins at the Furnas hydrobiology and hatchery station. In: PINTO-COELHO, R.M.; GIANI, A.; SPERLING, E. (eds.). Ecology and Human Impacts on lakes and reservoirs in Minas Gerais with special reference to future development and management strategies. Belo Horizonte: SEGRAC, 1994, 193p.

SANTOS-SILVA, E.N. Revisão das espécies do "complexo nordestinus" (Wright, 1935) do gênero Notodiaptomus Kiefer, 1936 (Copepoda: Calanoida: Diaptomidae). Tese de Doutorado, Universidade de São Paulo, USP, 2000.

SANTOS-WISNIEWSKI, M.J. Distribuição espacial e produção secundária da comunidade zooplanctônica do Reservatório de Barra Bonita - SP. 1998. 240 f. Tese (Doutorado em Ciências) - Centro de Ciências Biológicas e da Saúde, Universidade Federal de São Carlos, São Carlos, 1998.

SHANNON, C. E.; WEAVER, W. The mathematical theory of communication. University of Illinois, Press: Urbana, 1949. 
SILVA, W.M. Diversidade dos Cyclopoida (Copepoda, Crustacea) de água doce do estado de São Paulo: taxonomia, ecologia e genética. 154 f. Tese (Doutorado em Ciências) Centro de Ciências Biológicas e da Saúde, Universidade Federal de São Carlos, São Carlos, 2003

SIPAÚBA-TAVARES, L. H.; ROCHA, O. Produção de plâncton (Fitoplâncton e Zooplâncton) para alimentação de organismos aquáticos. São Carlos: RiMa, 2001, 106 p.

TER BRAAK, C. J. F.; ŠMILAUER, P. Canoco reference manual and CanoDraw for Windows user's guide: software for canonical community ordination. Microcomputer Power, Ithaca, NY, 2002.

THORNTON, K.W.; KIMMEL, L.B.; FONEST, E.P. Reservoir Limnology: ecological perspectives. New York: John Wiley, 246p., 1990.

TUNDISI, J.G.; MATSUMURA-TUNDISI, T.; CALIJURI, M.C.. Limnology and management of reservoirs in Brasil. In: STRAKRABA, M.; TUNDISI, J.G.; DUNCAN, A. (Ed.) Comparative reservoir limnology and water quality management. Dordrecht, Kluwer Academic Publishers. p. 25-55. 1993.

VALDERRAMA, J.C. The simultaneous analysis of nitrogen and total phosphorus in natural waters.Mar. Chem., Amsterdam, v.10, p. 109-122, 1981. 